\title{
Electrophysiology and Radio frequency Ablation - A Novel Method of Treatment in Cardiology
}

\section{Dr. Sujeeb Rajbhandari*}

Cardiac Electrophysiology and Radiofrequency Ablation (EPS \& RFA) is one of the newer modalities of treatment in the field of cardiology. This method of treatment is directed towards cardiac arrhythmias. Gone are the days when a doctor had to rely on medications to treat such conditions. It's an interventional technique which requires catheters, an EP lab system, cardiac catheterization laboratory and an Electrophysiologist.

\section{History}

William His, Jr, published a paper in 1843 describing centrally located pathway which eclectically connected atria to ventricles, which is now known as atrioventricular node. Electrophysiological study actually started during the beginning of $8^{\text {th }}$ decade and was limited to diagnosis only. The treatment for arrhythmias then was surgical. It was followed by direct current application via catheters. First catheter based ablation of atrioventricular junction in human was done in 1982. Radiofrequency ablation was introduced in the year 1987. The main advantage using this form of treatment was that it uses low energy burns so the tissue destruction and charring was less. The form of treatment has extended to microwave, cryothermy and pulsed ultrasound; all of which causes even less tissue destruction. Electrophysiology and radiofrequency ablation is most effective in treating arrhythmias due to atrioventricular nodal re-entry and those due to by pass tracts, with more sophisticated instruments and growing experience it is growing success in atrial tachycardias, recurrent atrial flutter and idiopathic ventricular tachycardias. 


\section{Skilled Manpower}

A cardiologist trained in electrophysiology and radiofrequency ablation for at least one year, preferably two years is required.

\section{Catheters}

Unlike coronary catheters, electrophysiology catheters do not have internal lumen. Previously they are made of woven Dacron which makes them stiff but they tend to soften and body temperature so allowing them to curve so making the manipulation easy. Nowadays they are made up of synthetic polyurethance blended material with internal stainless steel braiding. We commonly use two types, one for mapping and one for radiofrequency ablation. The tip is larger in the later type. Mapping catheter comes in deflectable type and non deflectable type while RF ablation catheter is deflectable.

\section{Vascular Access}

1. Left and right femoral veins

2. Right or left femoral artery

3. Right internal jugular vein

4. Other approaches:

a. Subclavian vein

b. Brachiocephalic vein

Leg veins are remarkably complaint and multiple catheters 2 to 3 can be inserted. The technique of vascular access is modified Seldinger technique which is fast, relatively painless and allows prompt catheter exchange.

\section{Uses of Cardiac Electro physiological Study}

\section{Diagnostic}

1. Sinus node dysfunction

2. Site of atrioventricular nodal block

3. Syncope of unknown etiology

4. Differentiation of VT and SVT in wide complex tachycardias,

5. Mechanism of SVT or VT

6. Map the origin of SVT or VT 


\section{Therapeutic}

1. To guide medical treatment for sustained VT, aborted Sudden cardiac death and SVT

2. Patient selection for Implantable Cardioverter Defibrillator

3. Test efficacy of devices for VT/VF

4. Patient selection for catheter ablation or surgical mode of therapy

5. Test efficacy of ablative or surgical treatment

\section{Interventional}

1. Slow pathway ablation for atrioventricular nodal re-entry

2. Accessory pathway ablation for WPW, Mahaim etc

3. Ablation of atrial tachycardia for and atrial flutter circuit

4. AV nodal modification for atrial fibrillation

\section{Risk Stratification}

1. Asymptomatic WPW

2. Post myocardial infarction

3. Non sustained VT

\section{Requirements}

1. Cardiac Catheterization Laboratory: For imaging purpose i.e, Digital substraction cardiography/fluoroscopy.

2. Stimulator: To stimulate atria or ventricle which is a part of an EP study with following features;

- it should be programmable

- Should be able to pace at various rates

- Should be able to pace from two different sites simultaneously

- Can be able to produce at least 3 extra premature stimuli coupled to a pacing Train (which consists of $8-10$ beats).

- Able to synchronize to patients spontaneous rhythm

3. Recorder; A computer (CPU + Monitor + Keyboard and mouse). To record and save intracardiac and surface ECG tracings for later viewing 
- Sweep speed (rate at which each beat/impulses is recorded) of $10-300 \mathrm{~mm} / \mathrm{sec}$

- Intracardiac ECGs should be displayed simultaneously with at least 3 -4 surface ECGs,

\section{Ablator:}

- RF energy of $200=1200 \mathrm{~K}_{\mathrm{Hz}}$

- Low voltage $(10=60$ volts $)$

- High frequency alternating current $\left(30 \mathrm{~K}_{\mathrm{HZ}}\right.$ to $\left.300 \mathrm{M}_{\mathrm{HZ}}\right)$

5. Junction Box: Consists of pairs of numbered multiple poles. It serves as an Interphase (junction) between the catheters and stimulator, recorder, ablator.

6. Interphase cable: It is the cable which connects catheter to junction box

\section{Catheter Placement}

- RA:

1. High posterolateral wall at junction with SVC

2. Right atrial appendage

\section{- LA:}

Indirectly via coronary sinus:

- In LAO view: Catheter curves towards left shoulder

- In RAO view: Catheter curves posteriorly

\section{- HIS Bundle:}

Catheter is entered into RV across tricuspid valve and withdrawn with slight clockwise torque. Initially large ventricular potential is recorded. Then small atrial signal appears before ventricular. When atrial and ventricular signal becomes equal in amplitude a bi or triphasic deflection appears in between which is "His" potential. Stable recording for several hours can be obtained without further manipulation.

- RV: Catheter is placed in the RV apex. 


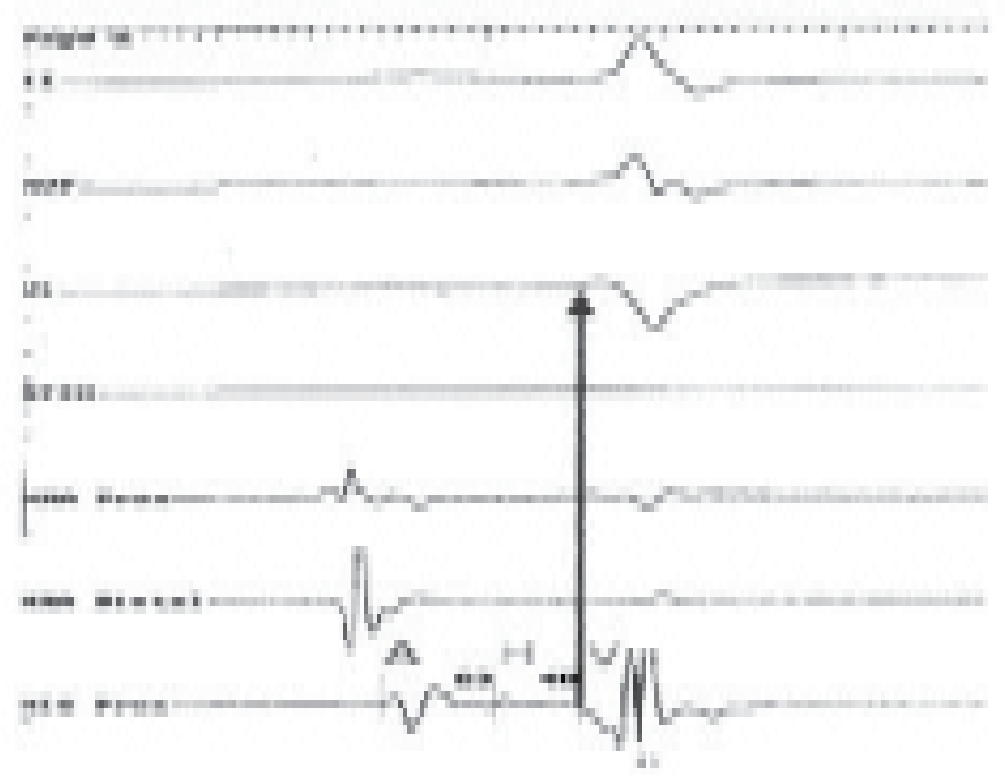

Fig: Top most 3 are ECG leads II, aVF and VI and the last three are intracardiac ECGs, namely High Right Atrium, His Distal and Ais proximal

\section{Complications}

In diagnostic studies: $1 \%$

In ablations: $2.5 \%$

1. Heamorrhage

2. Thrombo-embolism $-0.05 \%$

3. Phlebitis / DVT $=0.03 \%$

4. Arrhythmias: We have to induce arrhythmias as it is a part of testing protocol in EPS. Apart from the arrhythmias induced purposely, Atrial fibrillation is the most common arrhythmia encountered.

5. Arterio $=$ venous fistula

6. Cardiac tamponade $-0.08 \%$

\section{EPS \& RFA in Nepal}

This form of treatment is now available now in Shahid Gangalal National Heart Centre. With the help from electrophysiolgists from India and USA, we have performed EPS and Radiofrequency ablations in more than 100 patients with very good results. The patient population consisted mainly of supraventricular tachycardias. Recurrences were less than $3 \%$, no major complications occurred except for an femoral arterio-venous fistula which was later surgically repaired. 